\title{
Prediction of Remaining Useful Life for Lithium-Ionbattery with Multiple Health Indicators
}

\author{
ZHENG Yu-qiao ${ }^{1}$, CHEN Hong-jing ${ }^{2}$, SU Chun ${ }^{2}$
}

1. School of Mechanical and Electrical Engineering, Lanzhou University of Technology, Lanzhou 730050, China

2. School of Mechanical Engineering, Southeast University, Nanjing 211189, China

Corresponding author: SU Chun, Professor, phD; Tel: +86-13851875437; E-mail: suchun@ seu.edu.cn

\begin{abstract}
The remaining capacity can only be measured with offline method. This brings great challenge for the online prediction of Li-ion battery's RUL. A novel online prediction method for Li-ion battery's RUL was proposed, which is based on multiple health indicators (HIs) and can be derived from the batteries' historical operation data. Firstly, four indirect HIs were built according to the battery's operation current, voltage and temperature data respectively. On that basis, a generalized regression neural network (GRNN) was developed to estimate the battery's remaining capacity, and the non-linear autoregressive approach (NAR) was utilized to predict the battery's RUL based on the estimated capacity value. Furthermore, to reduce the interference, twice wavelet denoising were performed with different thresholds. A case study is conducted with a NASA battery dataset to demonstrate the effectiveness of the method. The result shows that the proposed method can obtain Li-ion batteries' RUL effectively.
\end{abstract}

Keywords: lithium-ion (Li-ion) battery; remaining useful life (RUL); health indicator (HI); generalized regression neural network (GRNN); non-linear autoregressive (NAR)

\section{Introduction}

Nowadays lithium-ion (Li-ion) battery has become a primary energy form for a variety of engineering equipments.Lithium-ion ( $\mathrm{Li}$-ion) battery is the key power unit for a variety of engineering equipments,including portable computer, electric automobile, satellite, and spacecraft, etc. However, with the increased cycles of charge-discharge, the battery's capacity will deteriorate gradually, and when the capacity is inferior to a given threshold, failure may occur. This phenomenon will reduce the equipment's reliability and even lead to catastrophic failures. Therefore, it is crucial to timely forecast Li-ion battery capacity as well as its remaining useful life (RUL).For Li-ion battery, its RUL can be defined as the number of remaining charge-discharge cycles before the battery capacity deteriorates to a predetermined failure threshold [1]. The prediction methods for battery RUL can be categorized into three types, i.e. model-based approach, data-driven approach and hybrid approach [2].Model-based approaches adopt mathematical representation or failure physics model to describe the degradation process of battery capacity, including electrochemical model [3-5], equivalent circuit model and empirical model [6], etc. Among them, electrochemical model aims to quantify the factors that affect battery performance and obtain the description of performance's evolution. Equivalent circuit model can simulate battery dynamic characteristics. However, considering the interactions among the various factors as well as the calculation of the parameters, it is not easy to establish a reliable and accurate prediction model for

Foundation item: Project 51965034/71671035 supported by the National Natural Science Foundation of China; Project ( 2018-RC-25) supported by Lanzhou city Innovation and Entrepreneurship of Lanzhou Technology Development Program. 
the above two approaches. Conversely, by fitting a large amount of degradation data, empirical model is easier to be established. Based on batteries' historical operational data, data-driven approach can extract the feature information and obtain the inherent degradation tendency. As a kind of data-driven approach, time series analysis has been widely used in RUL prediction, including auto-regressive (AR), auto-regressive and moving average (ARMA), auto-regressive integrated moving average (ARIMA) and the improved models [7-8]. In recent years, artificial intelligence (AI) algorithm becomes a hot point in data-driven approaches due to its powerful ability in self-learning and data mining. The frequently-used AI algorithms include artificial neural network (ANN) [9], support vector machine (SVM) [10, 11], relevance vector machine (RVM) [12], etc.

In most existing studies, the Li-ion battery's RUL is predicted with static capacity data. In fact, the battery's remaining capacity can only be measured when it is out of service. Furthermore, it also requires strict testing conditions and environment. Therefore, this kind of approach is difficult to be applied in engineering practice. To solve this problem, some other discriminant criteria can be used to estimate the battery's remaining capacity as well as its RUL, e.g. internal resistance [13, 14], incremental capacity analysis (ICA) [15, 16], open circuit voltage (OCV), entropy [17-18], etc. Here, we call them the health indicators (HIs).

In this work, on the basis of battery's operation data, a novel hybrid approach is proposed for estimating Li-ion battery RUL, and multiple HIs are adopted concurrently. Generally, there are three steps in it. Fig. 1 shows the basic procedure of the proposed approach.

Step 1: Calculating HIs. Based on the data, including battery current, voltage as well as working temperature, four types of HIs are built accordingly. They are calculated resistance (CR), rate of temperature change (TR), duration time of equal discharging voltage difference (DEDVD) and sample entropy of discharge voltage (SampEn) respectively.

Step 2: Estimating the remaining capacity. On that basis, a generalized regression neural network (GRNN) with cross-validation is applied to estimate the battery's remaining capacity. To reduce the interference and improve the accuracy of the estimation, the wavelet denoising is performed for two times and with different thresholds.

Step 3: RUL prediction. With the estimated remaining capacity, the battery's RUL is predicted via the non-linear autoregressive (NAR) method.

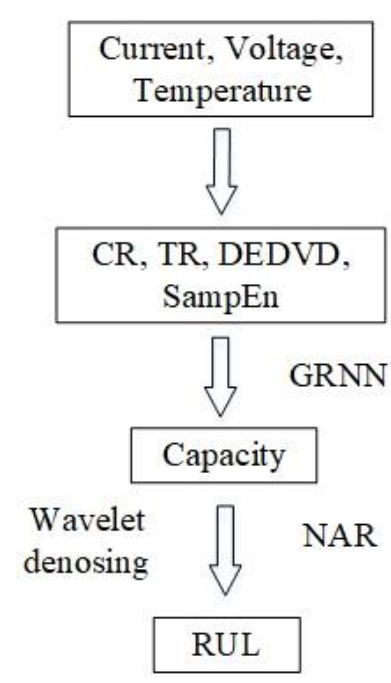

Fig. 1 Process of the proposed approach 
The remainder of this article is organized as follows. Section 2 introduces the four HIs respectively. Section 3 illustrates the basic theory of GRNN, NAR and wavelet denoising. Based on the Li-ion battery data from NASA, Section 4 conducts a case study of Li-ion battery RUL prediction, and the effectiveness of the proposed approach is illustrated. Conclusions and future works are given in Section 5.

\section{Health indicators}

To illustrate the discharging characteristics of the battery changing with the number of the charge cycles, in this study four HIs are extracted, they are CR, TR, DEDVD and SampEn respectively. The value of the above four HIs will change with the increase of cycles, and the corresponding calculation methods are described as the follows.

\subsection{Calculation of $\mathrm{CR}$}

For Li-ion battery, its internal resistance is closely related to the battery remaining capacity. CHANG Yang et al [19] found that the remaining capacity of Li-ion batteries is linearly related to the sum of their electrolyte impedance and the charge transfer impedance. Therefore, the internal resistance can be used to estimate the battery remaining capacity.

$\mathrm{CR}$ is the measured internal resistance, which can be obtained with battery terminal voltage and current during its operating state. Fig. 2 depicts the voltage and current's change at the instant of battery discharge. As shown in Fig. 2, when a constant DC current passes through the battery, the terminal voltage changes $\Delta U_{n}$ at the time $t_{1}$. At that time, the polarization effect has not occurred in the battery, therefore the voltage change is caused by ohm resistance. In this paper, the CR refers to the ohmic resistance $R_{n}$. According to Ohm's Law, it can be obtained as

$$
R_{n}=\frac{\Delta U_{n}}{I_{n}} \quad n=1,2, \ldots, N
$$

where $\Delta U_{n}$ is the voltage change caused by ohmic resistance of cycle $n ; I_{n}$ is the load current in cycle $n$; and $N$ is the total cycle life of the battery.

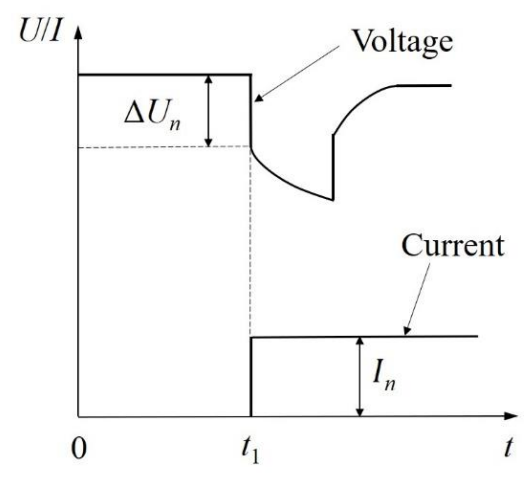

Fig. 2 Pulse discharge response of the battery

\subsection{Calculation of TR}

During the discharging process, the battery's internal temperature will change. Here we define $T R$ as the rate that the temperature changes in the discharge process. $T R$ will increase with the increase of cycles. In cycle $n, T R$ can be calculated as follows: 


$$
T R_{n}=\left(T P_{e}^{n}-T P_{s}^{n}\right) / t_{n} \quad n=1,2, \ldots, N
$$

where $T R_{n}$ denotes the rate of temperature change in cycle $n ; T P_{e}{ }^{n}$ is the end temperature of cycle $n ; T P_{s}{ }^{n}$ is the initial temperature of cycle $n$; and $t_{n}$ is the duration time of cycle $n$ 's discharge process.

\subsection{Calculation of DEDVD}

For Li-ion battery, the time for full discharge will be shortened gradually with the increase of the cycles. Therefore, the duration time of equal discharging voltage difference (DEDVD) will also be shortened with the increase of the cycles' number. For a constant voltage interval $\left[U_{L}, U_{H}\right]$. We define $t_{D}^{n}$ as the duration time in cycle $n$ when the voltage is reduced from $U_{H}$ to $U_{L}$, as shown in Fig. 3.

$$
t_{D}^{n}=\left|t_{H}^{n}-t_{L}^{n}\right| \quad n=1,2, \ldots, N
$$

where $t_{D}^{n}$ denotes the duration time of equal discharging voltage difference in cycle $n ; t_{H}^{n}$ denotes the moment that the discharge voltage is $U_{H}$ in cycle $n$; and $t_{L}^{n}$ denotes the moment that the discharge voltage is $U_{L}$ in cycle $n$.

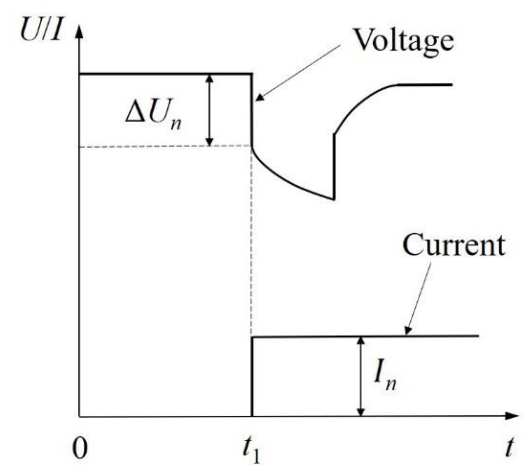

Fig. 3 Duration time of equal discharging voltage difference

\subsection{Calculation of SampEn}

Sample entropy (SampEn) was firstly proposed by Richman and Mooman to measure the complexity of time series [20]. In this study, we regard the discharging process as a time series, and the SampEn of discharge voltage is used as an $\mathrm{HI}$ for the Li-ion battery.

For a given embedding dimension $m$, tolerance $r$ as well as the number of data point $T$, we can obtain a time-series data set $X=\left\{x_{1}, x_{2}, x_{3}, \ldots, x_{T}\right\}$ with a constant time interval $\tau$. Here we define a template vector of length $m, \quad X_{m}(\alpha)=[x(\alpha), x(\alpha+1), \ldots, x(\alpha+m-1)] \alpha=1,2, \ldots, T-m+1$. The distance between two template vectors of $X_{m}(\alpha)$ and $X_{m}(\beta)$ is define as $d_{m}\left[X_{m}(\alpha), X_{m}(\beta)\right](\alpha \neq \beta)$. It can be any types of distance functions, including Euclidean distance, or Chebyshev distance.

$V^{m}(\alpha)$ is defined as the number of $d_{m}\left[X_{m}(\alpha), X_{m}(\beta)\right] \leq r(\alpha \neq \beta)$, and the definition of the function is as the follows [21]: 


$$
\begin{gathered}
B_{\alpha}^{m}(r)=\frac{1}{T-m+1} V^{m}(\alpha) \alpha=1,2, \ldots, T-m+1 \\
B^{m}(r)=\frac{1}{T-m} \sum_{\alpha=1}^{T-m} B_{\alpha}^{m}(r)
\end{gathered}
$$

where $B^{m}(r)$ represents the probability that two sequences will match for $m$ points. Similarly, we can obtain $B^{m+1}(r)$. Hence, the sample entropy can be defined as:

$$
\operatorname{SampEn}(r, m, T)=-\ln \left[\frac{B^{m+1}(r)}{B^{m}(r)}\right]
$$

\section{Methodology}

In this work, the battery's remaining capacity is estimated with the proposed HIs. On that basis, by analyzing the degradation trend of the remaining capacity, the battery's RUL is predicted.

As shown in Fig. 1, GRNN is used to establish the mapping relationship between the HIs and the battery's capacity, and hereafter to estimate the remaining capacity. Here, NAR model is used to demonstrate the degeneration of battery capacity. Moreover, wavelet denoising is applied to reduce the interference of the estimated capacity. Here, we illustrate the basic theories for GRNN, NAR and wavelet denoising as the follows.

\subsection{Theory of GRNN}

With a flexible network structure, GRNN model has strong ability for non-linear mapping. Therefore, it is suitable for solving non-linear problems [22].

Generally, there are four layers in GRNN, i.e. input layer, pattern layer, summation layer, and output layer, as shown in Fig. 4. The input of the network is $\mathbf{X}=\left[x_{1}, x_{2}, \ldots, x_{e}\right]^{T}$, and its output is $\mathbf{Y}=\left[y_{1}, y_{2}, \ldots, y_{k}\right]^{T} . d$ is the dimension of the input vector, $k$ is the dimension of the output sample, and $m$ is the number of the learning sample.

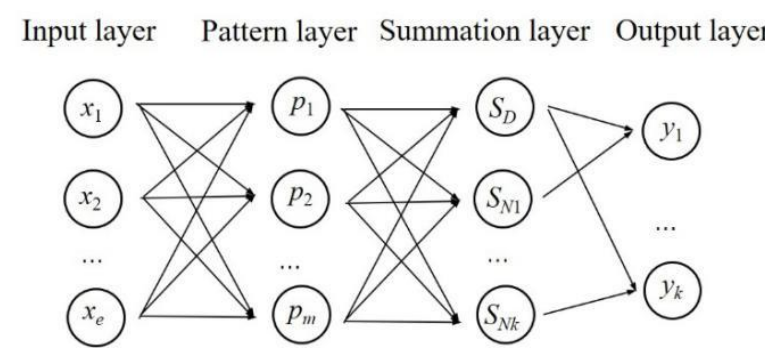

Fig. 4 Basic structure of the GRNN

(1) Input layer: It is responsible for the input data into the model. Among it, each neuron is a simple distribution unit, which will transfer the input variable directly to the pattern layer. In addition, the number of the input neurons in a GRN equals to the dimension of the input vector $e$.

(2) Pattern layer: The number of neurons equals to the number of learning sample $m$. Each neuron corresponds to a different sample, and the neurons transfer function can be calculated as : 


$$
p_{i}=\exp \left[-\frac{\left(\mathbf{X}-\mathbf{X}_{i}\right)^{T}\left(\mathbf{X}-\mathbf{X}_{i}\right)}{2 \sigma^{2}}\right] \quad i=1,2, \ldots, m
$$

where $\sigma$ is the smoothing parameter; $\mathbf{X}$ is the input variable; and $\mathbf{X}_{i}$ is the corresponding learning sample of neuron $i$.

(3) Summation layer: It sums the output of the pattern layer.

In this layer, two types of neurons are used for summation. The simple summation of the pattern outputs is determined with:

$$
S_{D}=\sum_{i=1}^{m} p_{i}
$$

And, the weighted summation of the pattern output can be determined by

$$
S_{N j}=\sum_{i=1}^{m} y_{i j} p_{i} \quad j=1,2, \ldots, k
$$

where $y_{i j}$ is the weight of the $i$-th neuron in the pattern layer, which is connected to the summation layer.

(4) Output layer: Based on the results calculated in the summation layer, the corresponding results will be sent to the output layer. The number of neurons is equal to the dimension $k$ of the output vector in the sample. The output of the neurons is calculated as follows:

$$
y_{j}=S_{N j} / S_{D} \quad j=1,2, \ldots, k
$$

where $y_{i}$ is the output of the $j$-th node in the output layer.

In this study, the cross-validation is used to gain the optimized parameters.

\subsection{Theory of NAR}

Here, by combining the variable's information during the early time, NAR model is used to describe the variable information at a particular time. It is a form of time series [23]:

$$
y(t)=f[y(t-1), y(t-2), \ldots, y(t-p)]
$$

where $p$ is the delay time, namely the order of the NN; and $f(\cdot)$ is the model of NAR.

The structure of NAR-DNN is shown as in Fig. 5. It is consisted of four layers, i.e. input layer, hidden layer, output layer as well as the delay function.

In Fig.5, $x_{u}(1 \leq u \leq p)$ is the input; $y(k)$ is the output; $W_{1}$ is the connection weight between the input layer and the hidden layer; $W_{2}$ is the connection weight between the hidden layer and the output layer; and $p$ is the delay time of the output. 


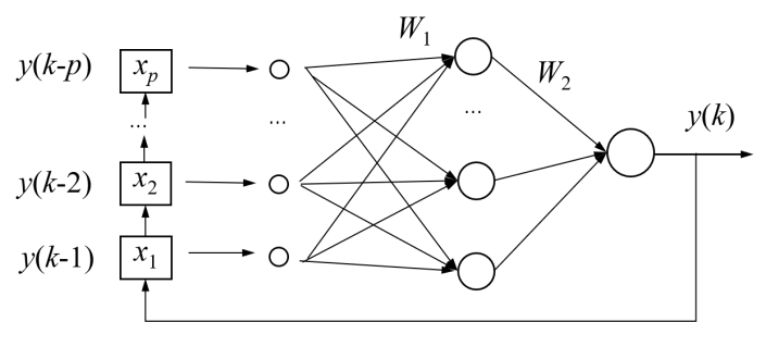

Fig. 5 Structure of NAR-DNN

The output of the node $v$ in the hidden layer is

$$
h_{v}=g_{1}\left(\sum_{u=1}^{p} w_{u v} x_{u}+c_{v}\right) \quad v=1,2, \ldots, V
$$

where $g_{1}(\cdot)$ is the activation function of the hidden nodes; $V$ is the number of the hidden nodes; $x_{u}$ is the $u$-th delay output of the output signal $y ; \quad w_{u v}$ is the connection weight between the hidden node $u$ and the delay node $v$; and $c_{v}$ is the $v$-th neural threshold of the hidden layer.

The output of NAR is

$$
y=g_{2}\left(\sum_{v=1}^{V} w_{v} h_{v}+d\right)
$$

where $w_{v}$ is the connection weight between the hidden node $v$ and the output node; and $d$ is the neural threshold of the output layer.

\subsection{Wavelet denoising}

Wavelet analysis is a kind of method for signal analysis, which can separate the noise signals and extract effective data from the noise-mixed data. In wavelet analysis, the data can be decomposed into two parts with the expansion and translation of the mother wavelet, i.e. approximate part and detail part [24].

Let $H(x)$ denote the measured signal mixed with noise, the wavelet transform with the mother wavelet $\psi(x)$ can be expressed as:

$$
c(a, b)=\left\langle H(x), \psi_{a, b}(x)\right\rangle=\frac{1}{\sqrt{|a|}} \int_{-\infty}^{+\infty} H(x) \psi\left(\frac{x-b}{a}\right)
$$

where $a$ and $b$ are the expansion and translation parameters of the mother wavelet respectively; and $c(a, b)$ is the wavelet coefficient of $H(x)$.

During the process of wavelet denoising, the selection of wavelet denoising threshold is closely related to the consequence of noise reduction. In this study, a twice denoising method with different thresholds is proposed.

The first denoising threshold is made by the Sqtwolog method [25]:

$$
\lambda_{s q t}=\sqrt{2 \log N}
$$

The second denoising threshold is finished by the minimax method [25]: 


$$
\lambda_{\min }=0.3936+0.1829 \log _{2}^{N}
$$

where $N$ is the number of signal $H(x)$, and in this paper, it equals to the cycle life of the battery.

The threshold of the above two methods are different. Normally, the threshold of the Sqtwolog method is larger than that of the minimax method. Therefore, the first wavelet denoising can remove the lager noise signal, while the second wavelet denoising can remove the smaller noise signal. By using twice wavelet denoising with different thresholds, the interference among the measured data can be reduced effectively.

\subsection{Implementation of the proposed approach}

Here, the training batteries are used to establish the mapping relationship between the HIs and the battery capacity. The basic steps for estimating battery RUL are described as follows:

(1) Based on the historical operation data, the HIs of the training batteries and testing batteries are calculated respectively.

(2) By using the training batteries, train a GRNN model to establish the mapping relationship between the HIs and the battery's capacity.

(3) Based on the well-trained GRNN model and the HIs data of testing batteries in the previous cycles, the capacity of the testing batteries is estimated accordingly.

(4) The wavelet denoising is applied to deduce the noising of the estimated capacity.

(5) With the established capacity sequence and by using the NAR model, the remaining capacity and RUL of the testing battery can be predicted

\section{Prognostics Experiment}

\subsection{Experiment Data and the Procedure}

In this section, a case study is conducted to demonstrate the effectiveness and efficiency of the proposed approach, where the Li-ion battery data come from the data repository of the NASA Ames Prognostics Center of Excellence (PCoE) [26]. Here, three groups of batteries are taken as examples, i.e. B05, B06 and B07. Among them, B05 and B06 are used as the training batteries, and B07 is used as the target battery. The experimental parameters of the discharge process are listed as in Table 1.

Table 1 Discharging parameters of the NASA batteries

\begin{tabular}{cccccc}
\hline Battery No. & $T /{ }^{\circ} \mathrm{C}$ & $I_{\text {dis }} / \mathrm{A}$ & $V_{\text {up }} / \mathrm{V}$ & $V_{\text {low }} / \mathrm{V}$ & $C_{\text {new }} / \mathrm{Ah}$ \\
\hline B05 & 24 & 2 & 4.2 & 2.7 & 1.86 \\
B06 & 24 & 2 & 4.2 & 2.5 & 2.04 \\
B07 & 24 & 2 & 4.2 & 2.2 & 1.89 \\
\hline
\end{tabular}

The capacity's degradation trends with the cycles are shown as in Fig. 6. Obviously, the battery capacity shows a non-monotonic decline with the increase of the cycles. Meanwhile, the degradation is accompanied with the phenomena of capacity self-regeneration as well as local fluctuations. Therefore, it is essential to develop an effective prognostic approach to accurately estimate the capacity and predict its RUL by overcoming 
the uncertainty fluctuation.

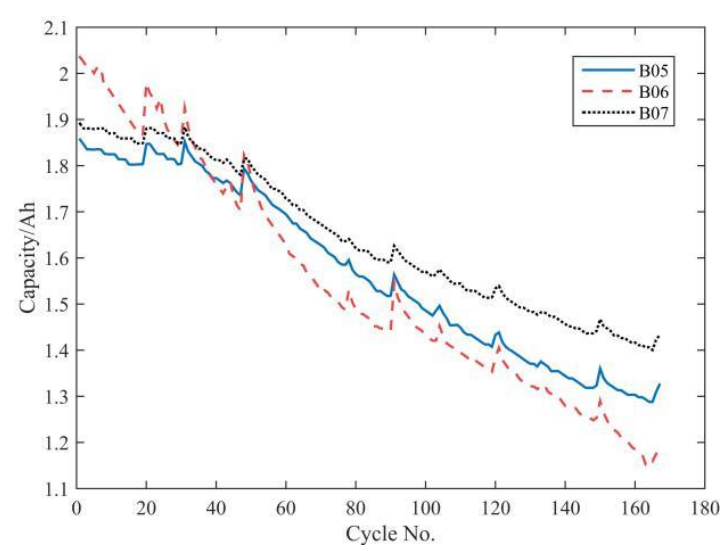

Fig. 6 Capacity degradation of tested batteries

\subsection{Calculating HIs}

In this section, by using the batteries' historical operation data (including current, voltage and temperature) during the discharging process, we calculate the HIs for batteries of B05, B06 and B07 respectively. Fig. 7 shows the variation of the four HIs with the increase of cycles respectively.

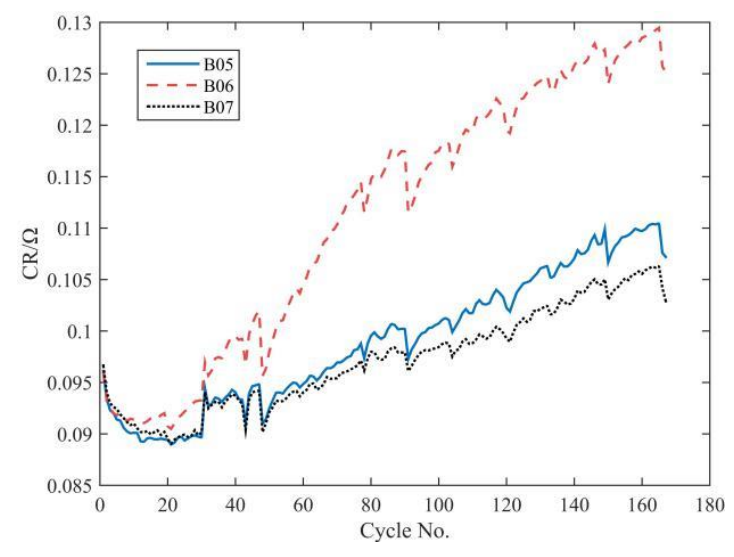

(a) Variations of $\mathrm{CR}$ 


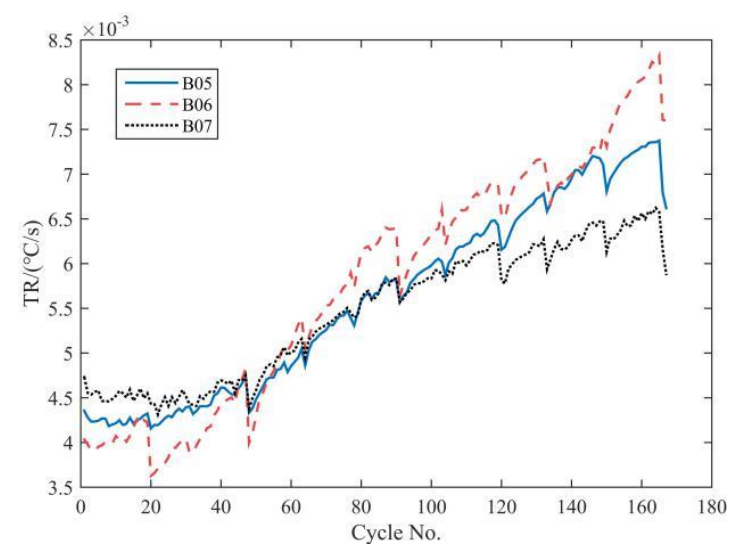

(b) Variations of TR

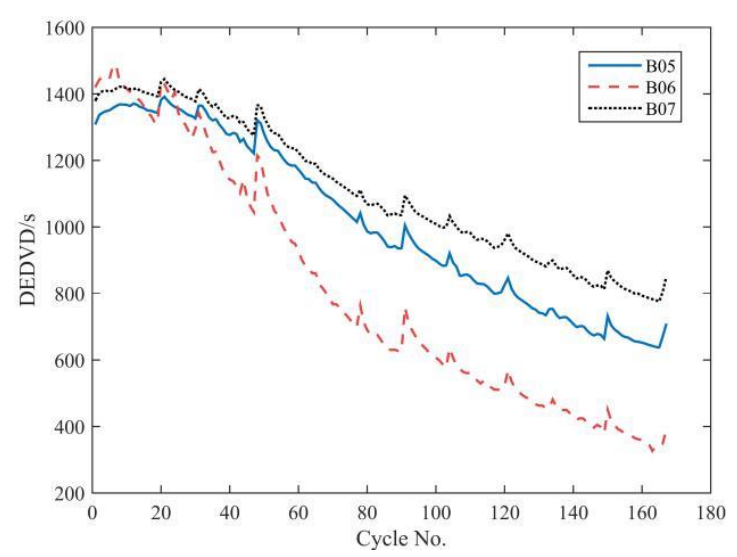

(c) Variations of DEDVD

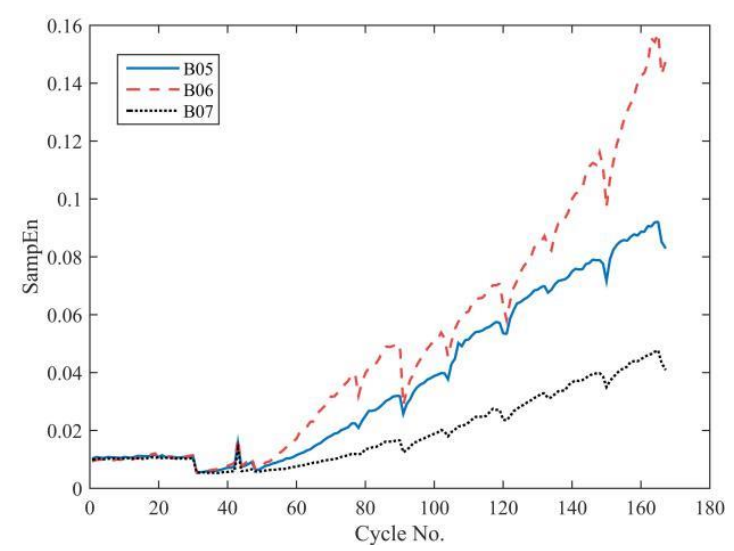

(d) Variations of SampEn

Fig. 7 Variations of the four HIs changing with the charging cycles

Next, we calculate the Pearson correlation coefficient between the four HIs and the original capacity of Li-ion batteries, as shown in Table 2. Obviously, the HIs have a close correlation with the capacity. Furthermore, the DEDVD has a negative correlation with the capacity, while the other three HIs are in positive correlation.

Table 2 Correlation coefficient between HIs and capacity 


\begin{tabular}{cccc}
\hline Battery No. & B05 & B06 & B07 \\
\hline CR & -0.9791 & -0.9893 & -0.9658 \\
TR & -0.9947 & -0.9819 & -0.9901 \\
DEDVD & 0.9988 & 0.9957 & 0.9990 \\
SampEn & -0.9522 & -0.8936 & -0.8876 \\
\hline
\end{tabular}

\subsection{Establishing GRNN model}

With the obtained HIs data, GRNN model is used to train and establish the mapping relationship between the HIs (input) and the capacity (output). In this study, the data of B05 and B06 batteries are used for training. On that basis, the well-trained GRNN model is used to estimate B07's capacity with B07's HIs. The predicted results are shown in Fig. 8.

As shown in Fig. 8, the proposed method has a relatively high prediction accuracy concerned with the capacity in the long-term trend. However, the predicted result is not so good for the first 40 points. The reason is that the HIs is not so stable during the early stage. Therefore, the first 40 points can be removed to get a more accurate prediction result.

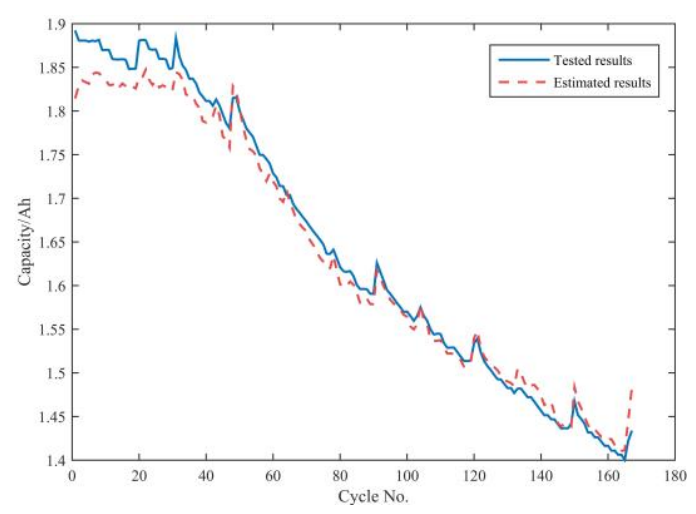

Fig. 8 The estimated and tested capacity of B07

Moreover, the indicators, including root means square error (RMSE), the maximum estimation error and the maximum covariance (cov) value, are utilized to evaluate the accuracy of the estimation results, as shown in Table 3. Obviously, the capacity estimation is with high accuracy.

Table 3 Accuracy analysis of the capacity estimation

\begin{tabular}{ccc}
\hline Indicators & All points & Remove the first 40 points \\
\hline RMSE & 0.2002 & 0.0140 \\
Max error & 0.0823 & 0.0267 \\
Max cov & 0.0259 & 0.0110 \\
\hline
\end{tabular}




\subsection{RUL prediction}

To remove the influence of self-regeneration phenomena and local fluctuations, a twice wavelet denoising is implemented with different thresholds. The result is shown as in Fig. 9.

In this case, the threshold of Sqtwolog method is larger than that of minimax method. As shown in Fig. 9, the first denoising can only eliminate the large noise signal, and the second denoising can eliminate the smaller noise signal. Thus, with the proposed twice wavelet denoising, the noise can be eliminated more thoroughly and a more accurate prediction can be obtained.

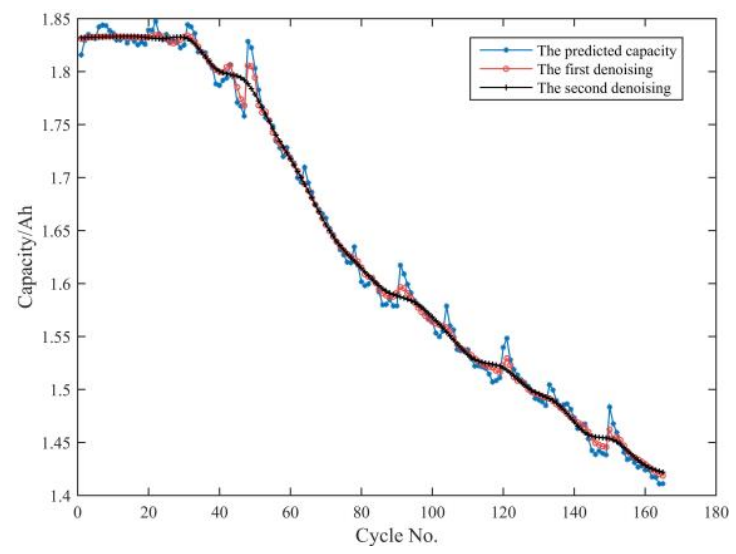

Fig. 9 The predicted capacity of B07

Based on the historical operation data, we can estimate the remaining capacity for each past cycle with the above steps. In addition, by selecting the obtained data points as the input set, the future degraded capacity can be predicted with the NAR model. On that basis, we can obtain the corresponding value of the RUL. Here, the number of the hidden layer is set as 1 , the delay time is 20 . It is supposed that the failure threshold of the battery capacity is $1.45 \mathrm{Ah}$, and the total cycle life of B07 is 142 . Here, we predict the B07's RUL when the operated cycles $(M)$ are 110,120, 130 respectively. In addition, considering that there is randomness in the training model, the predicted RUL will change with the experiments, thus we repeat the experiments for 100 times. Fig. 10 and Table 4 show some of the predicted results.

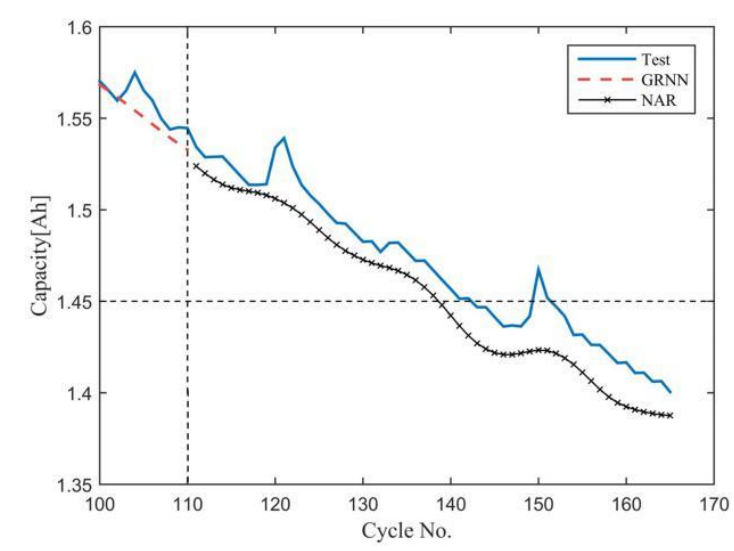

(a) $M=110$ 




(b) $M=120$

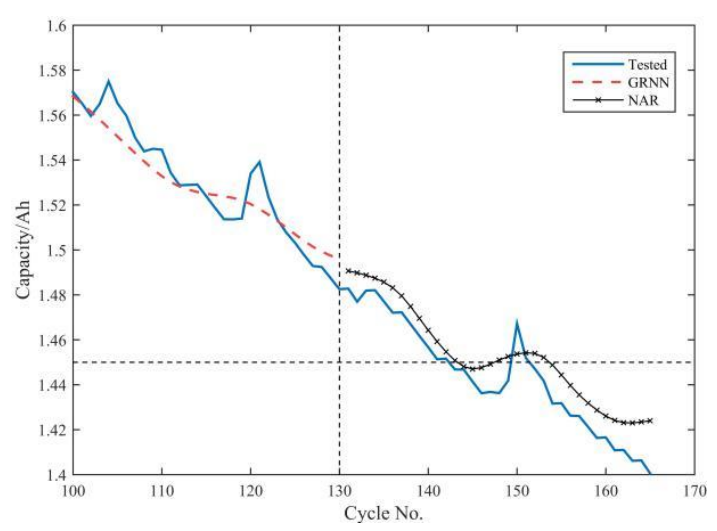

(c) $M=130$

Fig. 10 Predictions for B07's RUL under different $M$

Table 4 The predicted results of B07's RUL

\begin{tabular}{ccccc}
\hline$M$ & Actual EOL & Actual RUL & Predicted RUL & Percentage Error/\% \\
\hline 110 & 142 & 32 & 28 & 14.3 \\
120 & 142 & 22 & 23 & 4.3 \\
130 & 142 & 12 & 14 & 14.3 \\
\hline
\end{tabular}

Set the range of the 100 predicted results as the RUL uncertainty bound (RUB). For the battery of B07, the RUB is shown as in Table 5.

Table 5 The RUB of B07 battery

\begin{tabular}{lllll}
\hline$M$ & Actual EOL & Actual RUL & RUB & Range \\
\hline
\end{tabular}




\begin{tabular}{lllll}
\hline 110 & 142 & 32 & {$[28,55]$} & 27 \\
120 & 142 & 22 & {$[23,45]$} & 22 \\
130 & 142 & 12 & {$[14,35]$} & 21 \\
\hline
\end{tabular}

With the above predicted results, we can conclude that the proposed methods can obtain the decline tendency of the Li-ion battery capacity with a high accuracy. However, the RUL's predicted result is not so stable. The possible reason is that the tested life cycle of the battery is not long enough. Therefore, the training data are not sufficient. Furthermore, considering that the capacity change during each cycle is too small, and a small error in capacity prediction may lead to a larger error in the RUL's prediction. It can also be found that the range of RUB will be narrowed down with the increase of the input cycles. It shows that the RUL prediction is sensitive to the number of input data. Therefore, by increasing the number of the training samples, the stability and robustness of the predictions can be improved.

ARIMA model is also widely uses in battery RUL prediction. To compare the characteristics of different prediction models, here we make a comparison for RUL prediction with the ARIMA model. For the same input, the ARIMA method has a unique prediction result. Thus, the prediction results do not have a RUB. The RUL predicted results with ARIMA model are shown as in Table 6.

Table 6 Predicted RUL results based on ARIMA model

\begin{tabular}{ccccc}
\hline$M$ & Actual RUL & Predicted RUL & Error & Percentage Error/\% \\
\hline 110 & 32 & 38 & 6 & 18.9 \\
120 & 22 & 28 & 6 & 27.3 \\
130 & 12 & 18 & 6 & 50.0 \\
\hline
\end{tabular}

By comparing the three predicted results of different $M$, it can be found that for different $M$, the errors keep the same. It means that the prediction accuracy is not sensitive to the number of input cycles.

Comparing Table 4 and Table 6, it can be found that the predicted results of NAR model are more accurate than the results of ARIMA model. However, the predicted results are not as stable as the results of ARIMA model. Furthermore, NAR model is sensitive to the number of the input data, the proposed NAR method is more suitable for large data set.

\section{Conclusions}

An innovative hybrid data-driven method is proposed to predict Li-ion battery's RUL, which is based on multiple HIs, including CR, TR, DEDVD and SampEn. Furthermore, the GRNN model, NAR model and twice wavelet denoising are integrated. Case study shows that the proposed method can achieve Li-ion battery's RUL prediction with a high accuracy. Compared with ARIMA model, NAR model is more sensitive to the size of training samples. Furthermore, the proposed method is established on the basis of battery historical data, it can overcome the limitation of online capacity measurement approaches. Meanwhile, the proposed RUL prediction 
method does not concern the physical and chemical reactions in the battery, therefore it is well suitable for other kinds of batteries.

\section{References}

[1] YANG Chang, FANG Hua-jing, ZHANG Yong. A new hybrid method for the prediction of the remaining useful life of a lithium-ion battery [J]. Applied Energy,2017, 206: 1564-1578.

[2] HUANG Wen-qiang, LIU Gui-hua, LIU Peng. Equilibrium concentration of lithium ion in sodium aluminate solution [J]. Journal of Central South University, 2019, 26:304-311.

[3] CHAO, Lyu. LAI Qingzhi. GE A lead-acid battery's remaining useful life prediction by using electrochemical model in the particle filtering framework [J]. Energy, 2017,120:975-984.

[4] WAAG, Wladislaw, KAEBITZ, STEFAN, tefan, Experimental investigation of the lithium-ion battery impedance characteristic at various conditions and aging states and its influence on the application $[\mathrm{J}]$. Applied Energy, 2013,102: 885-897.

[5] PRASAD, Githin K, RAHN, Christopher D. Model based identification of aging parameters in lithium ion batteries [J]. Power Sources, 2013, $232: 79-85$.

[6] HU, Xiao-song, LI Sheng-bo, PENG Hui. A comparative study of equivalent circuit models for Li-ion batteries [J]. Power Sources , 2012, $198: 359-367$.

[7] YU Jin-song, TANG, Wei-qi, TANG, Di-yin. An approach to predicpt discharge voltage of lithium-ion batteries under dynamic loading conditions [J].Journal of Ambient Intelligence and Humanized Computing, 2019, 10:923-936

[8] TANG Sheng-jin, YU Chuan-qiang, FENG Yong-bao.Remaining useful life estimation based on Wiener degradation processes with random failure threshold [J]. Journal of Central South University, 2016, 23:2230-2241.

[9] XING Yin-jiao, MA EdenW.M., TSUI Kwork-Leung. An ensemble model for predicting the remaining useful performance of lithium-ion batteries [J]. Microelectron Reliability, 2013, 53(6): 811-820.

[10] LIU Da-tong, LUO Yue, LIU Jie. Lithium-ion battery remaining useful life estimation based on fusion nonlinear degradation AR model and RPF algorithm [J]. Neural Computing and Applications, 2014, 25(3-4): 557-572.

[11] ZHOU Ya-peng, Huang Miao-hua. Lithium-ion batteries remaining useful life prediction based on a mixture of empirical mode decomposition and ARIMA model [J]. Microelectronics Reliability, 2016(65): 625-273.

[12] LONG Bing, XIAN Wei-ming, LIU Jian. An improved autoregressive model by particle swarm optimization for prognostics of lithium-ion batteries [J]. Microelectronics Reliability, 2013, 53(6): 821-831.

[13] WU Ji, ZHANG Chen-bing, CHEN Zong-hai. An online method for lithium-ion battery remaining useful life estimation using importance sampling and neural networks [J]. Applied Energy, 2016(173): 134-140. 
[14] SBARUFATTI Claudio, CORBETTA Matteo, GIGLIO Marco, et al. Adaptive prognosis of lithium-ion batteries based on the combination of particle filters and radial basis function neural networks [J]. Journal of Power Sources, 2017, 344: 128-140.

[15] PATIL Meru. A, TAGADE Piyush, HARIHARAN Krishnan. S, et al. A novel multistage support vector machine based approach for Li ion battery remaining useful life estimation [J]. Applied Energy, 2015,159: 285-297.

[16] ZHENG Xiu-juan, FANG Hua-jing. An integrated unscented kalman filter and relevance vector regression approach for lithium-ion battery remaining useful life and short-term capacity prediction [J]. Reliability Engineering System Safety, 2015,144: 74-82.

[17] FENG Jing, KVAM Paul, TANG Yan-zhen. Remaining useful lifetime prediction based on the damage mar -er bivariate degradation model: A case study on lithium-ion batteries used in electric vehicles [J]. Engineering Failure Analysis, 2016,70: 323-342.

[18] SONG Yu-chen, LIU Da-tong, YANG Chen, et al. Data-driven hybrid remaining useful life estimation approach for spacecraft lithium-ion battery [J]. Microelectronics Reliability, 2017,75: 142-153.

[19] CHANG Yang, FANG Hua-jing, ZHANG Yong. A new hybrid method for the prediction of the remaining useful life of a lithium-ion battery [J]. Applied Energy, 2017,206: 1564-1578.

[20] KIM Jonghoon, LEE Seongjun, CHO B. H. Complementary cooperation algorithm based on DEKF combined with pattern recognition for SOC/capacity estimation and SOH prediction [J]. IEEE Transactions on Power Electronics, 2011, 27(1): 436-451.

[21] WENG Cai-hao, CUI Yu-jia, SUN Jing. On-board state of health monitoring of lithium-ion batteries using incremental capacity analysis with support vector regression [J]. Journal of Power Sources, 2013, 235(4): $36-44$.

[22] WENG Cai-hao, SUN Jing, Peng Huei. Model parametrization and adaptation based on the invariance of support vectors with applications to battery state-of-health monitoring [J]. IEEE Transactions on Vehicular Technology, 2015, 64(9):3908-3917.

[23] WIDODO Achmad, SHIM Min-Chan, CAESARENDRA Wahyu, et al. Intelligent prognostics for battery health monitoring based on sample entropy [J]. Expert Systems with Applications, 2011, 38(9):11763-11769.

[24] HU Xiao-song, LI Shengbo-ben, JIA Zhen-zhong, et al. Enhanced sample entropy-based health management of Li-ion battery for electrified vehicles [J]. Energy, 2014,64: 953-960.

[25] CHEN Lu-ping,XU Liang-jun, ZHOU, Yi-lin.Novel approach for lithium-ion battery on-Line remaining useful life prediction based on permutation entropy [J]. Energies, 2018, 11(4):820.

[26] B. Sahaand, K. Goebel. Battery Data Set, NASA ames prognostics data repository, 2007, NASA Ames Research Center. 